\title{
Light confinement in a low-refraction-index microcavity bonded on a silicon substrate: supplementary material
}

\author{
LI WANG ${ }^{1}$, SHU-XIN ZHANG ${ }^{1}$, QINGHAI SONG ${ }^{2}$, QIHUANG GONG ${ }^{1,3}$, AND YUN- \\ FENG XIAO ${ }^{1,3 *}$
}

${ }^{1}$ State Key Laboratory for Mesoscopic Physics and School of Physics, Peking University; Collaborative Innovation Center of Quantum Matter, Beijing 100871, P. R. China

${ }^{2}$ Integrated Nanoscience Lab, Department of Electrical and Information Engineering, Harbin Institute of Technology, Shenzhen, China, 518055

${ }^{3}$ Collaborative Innovation Center of Extreme Optics, Taiyuan 030006, Shanxi, P. R. of China

*Corresponding author: yfxiao@pku.edu.cn

Published 23 August 2016

This document provides supplementary information to "Light confinement in a low-refraction-index microcavity bonded on a silicon substrate," http://dx.doi.org/10.1364/optica.3.000937. @ 2016 Optical Society of America

http://dx.doi.org/10.1364/optica.3.000937.s001

\section{Geometry of the cross section}

Figure S1 shows the geometry of the cross section of the toroidal part taken by an angle of $56^{\circ}$ with the horizontal substrate. The boundary of the toroidal part is nearly a perfect circle, shown by the red dashed line in Fig. S1, which looks like an ellipse because of the oblique view. The disk part is connected with the substrate due to the second pulse of $\mathrm{CO}_{2}$ laser which slightly melts the microcavity.

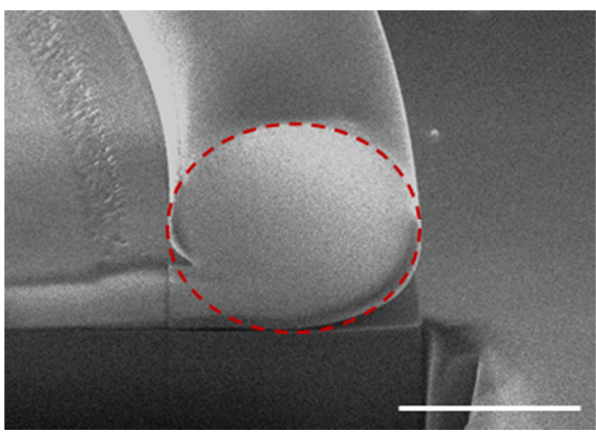

Fig. S1. Scanning electron microscope (SEM) image of the cross section of the toroidal part. It is an oblique view taken by an angle of $56^{\circ}$ with the horizontal substrate. The red dashed line show the boundary of a perfect circle. Scale bar: $5 \mu \mathrm{m}$.

\section{Effects of circular cross section}

The circular cross section of donut-shaped microcavity remarkably reduces the electric field distribution area in the vertical direction compared with the traditional squared cross section, resulting in a better light confinement of silica, as shown in
Fig. S2. With obvious field leakage into the silicon substrate, the simulated $Q$ factor of the squared one is only 5005 (see Fig. S2(a)), while the same sized circular one supports a fundamental mode with a $Q$ factor of 53502 (see Fig. S2(b)) which is more than 10 times higher. The shape induced difference could be even more tremendous as the minor diameter of the cross section increases.
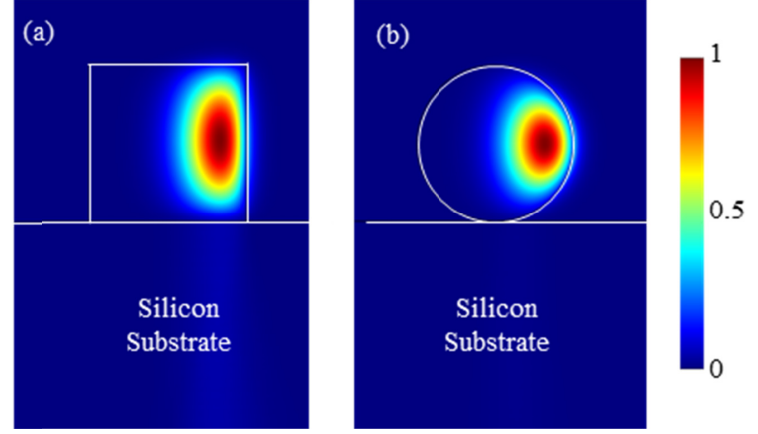

Fig. S2. Numerical simulation results with squared (a) and circular (b) cross section silica bonding to silicon substrate in $1550 \mathrm{~nm}$ wavelength band by finite element method. Both the side length of the square and diameter of the circle are $6 \mathrm{um}$. The principal diameter of the microcavity is $83 \mu \mathrm{m}$.

\section{Effects of the disk part}



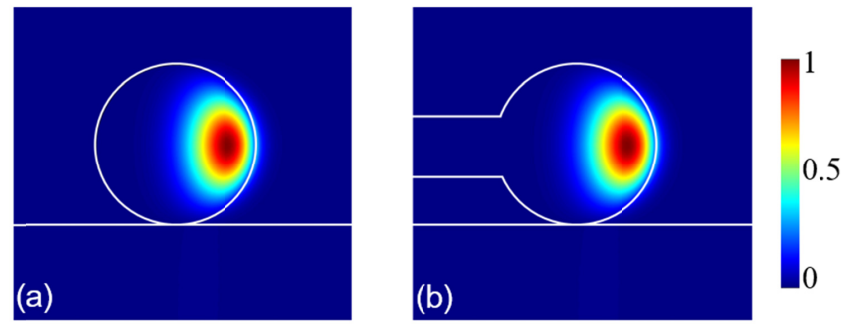

Fig. S3. Numerical simulation results of the microcavities without (a) and with (b) the disk part. The principal (minor) diameter of the microcavity is $80(6) \mu \mathrm{m}$.

The simulation results show that only few field is distributed in the left part of the cross section of the donut- shaped microcavity. Thus, when an extra disk part is connected to the inner (left) of the toroidal part, it hardly affects the resonant mode. The $Q$ factors of the mode in Figs. S3(a) and S3(b) are 50969 and 51044, which are almost the same.

\section{Effects of the substrate on TE mode}

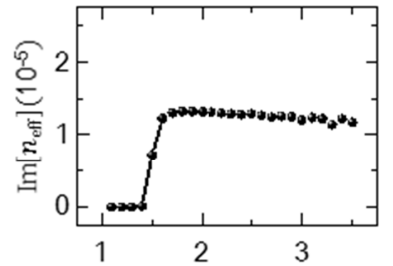

(a) Substrate refraction index

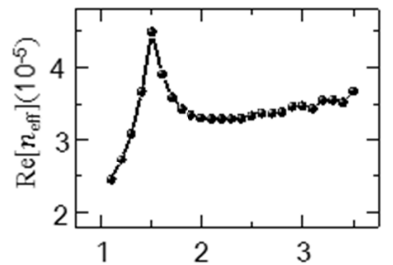

(b) Substrate refraction index
Fig. S4. The $\operatorname{Im}\left(n_{\text {eff }}\right)$ (a) and $\operatorname{Re}\left(n_{\text {eff }}\right)$ (b) versus the substrate refraction index $n_{\text {sub }}$, while the donut-shaped microcavity refraction index keeps unchanged. A value of 1.36500 is subtracted from $\operatorname{Re}\left(n_{\text {eff }}\right)$. The minor (principal) diameter of the microcavity is $6(80) \mu \mathrm{m}$ and the main polarization is TE.

Figures S4(a) and S4(b) show $\operatorname{Im}\left(n_{\text {eff }}\right)$ and $\operatorname{Re}\left(n_{\text {eff }}\right)$ of TE polarized mode depending on the substrate refraction index while Figs. 1(b) and 1 (c) in the main text are that of TM polarized mode. As shown in Fig. S4(a), $\operatorname{Im}\left(n_{\text {eff }}\right)$ has a sharp increase when $n_{\text {sub }}$ is around 1.5. For $n_{\text {sub }}$ larger than 1.5, $\operatorname{Im}\left(n_{\text {eff }}\right)$ maintains a stable value for $n_{\text {sub }}$ with the increase of $n_{\text {sub }}$, which means that the mode energy distributed in the silicon keeps the same, increasing its contribution to $\operatorname{Re}\left(n_{\text {eff }}\right)$. Thus, the curve of $\operatorname{Re}\left(n_{\text {eff }}\right)$ has a slowly rising tail for TE polarization in contrast with the fallen tail of TM polarization in the main text.

\section{Electric field distributions of modes with different orders}

Compared with the fundamental mode in Fig. S5(a), higher-order modes in Figs. S5(b)-S5(d) have larger field distribution areas in either azimuthal or radial direction, which accounts for the stronger energy leakages and lower $Q$ factors. In particular, the higher-order radial mode (Fig. S5(d)) suffers a stronger effect than azimuthal modes (Figs. S5(b), S5(c)). Ignoring the difference of polarization, Figs. S5(a)-S5(d) correspond to modes $\mathbf{T E}_{229,229}^{1}$ $\mathrm{TE}_{230,230}^{1}, \mathrm{TM}_{229,229}^{1}, \mathrm{TM}_{230,230}^{1}$, modes $\mathrm{TE}_{224,223}^{1}, \mathrm{TE}_{225,224}^{1}$, $\mathbf{T M}_{224,223}^{1}, \mathrm{TM}_{225,224}^{1}$, modes $\mathrm{TE}_{219,217}^{1}, \mathrm{TE}_{220,218}^{1}, \mathbf{T M}_{220,218}^{1}$, $\mathbf{T} \mathbf{M}_{221,219}^{1}$ and modes $\mathbf{T} \mathbf{M}_{223,223}^{2}, \mathbf{T M}_{224,224}^{2}$ in Fig. 2 (see main text).
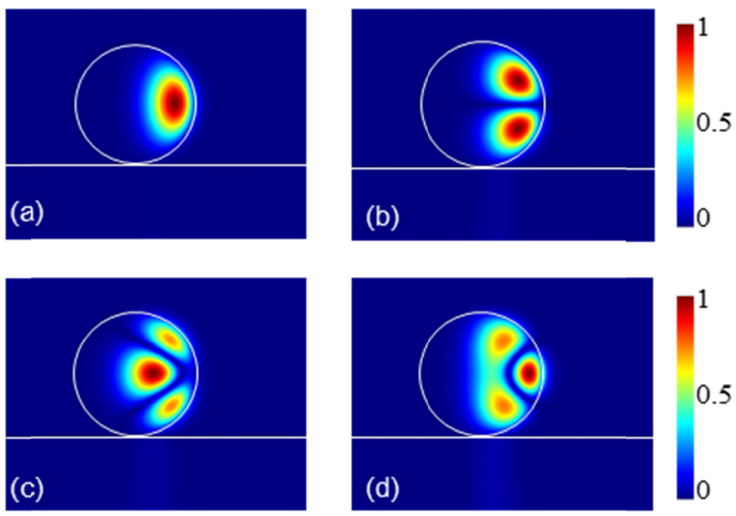

Fig. S5. Electric field distributions of modes with different mode orders. (a) Fundamental mode. (b), (c) Second and third order modes in azimuthal direction. (d) Second order mode in radial direction. The principal diameter of the microcavity is $80 \mu \mathrm{m}$.

\section{TE-TM $Q$ factor crossing}

Numerical simulation indicates that the $Q$ factors of both fundamental TE and TM modes increase exponentially with the increasing of minor diameter. The slope of TE mode is slightly steeper than TM mode, resulting in a crossing point around $d=6.5$ $\mu \mathrm{m}$. When $d$ is smaller (larger) than $6.5 \mu \mathrm{m}$, the TM (TE) fundamental mode possesses the highest $Q$ factor.

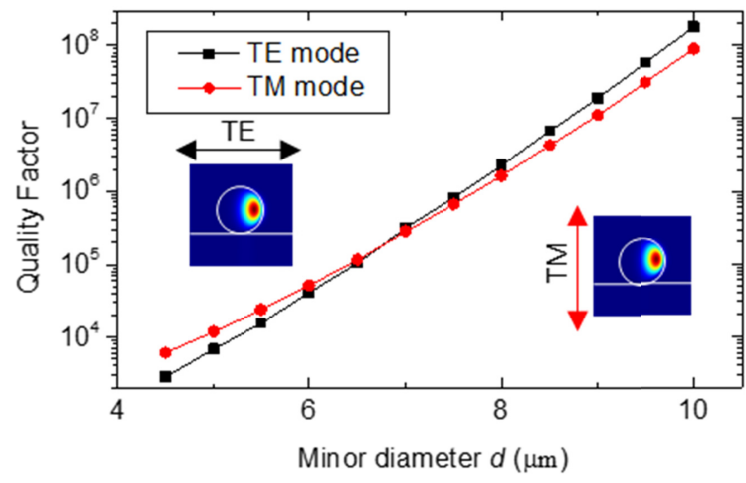

Fig. S6. Simulated $Q$ factor of fundamental TE (TM) mode versus minor diameter $d$ in communication band. The arrows show the principal polarization component of electric field. The principal diameter of the microcavity is $80 \mu \mathrm{m}$.

\section{Effects of cross-section geometric deformation}

Figure S7 shows the effect of cross-section geometry deformation on the $Q$ factors of fundamental TE and TM modes. Here X-axis (Yaxis) is defined as the diameter of the cross section in the horizontal (vertical) direction, and the diameter of the original circular cross section is $6 \mu \mathrm{m}$. When X-axis or Y-axis is stretched (compressed), the $Q$ factors of the fundamental modes increase (decrease). Comparing the curves in Fig. S7(a) with that in Fig. S7(b), the variation of $Q$ factor along with the change of X-axis is smaller. Moreover, the $Q$ factor of TM mode is particularly stable with the change of X-axis. We can also easily figure out that the $Q$ factor decreases by less than one order of magnitude even for twenty percent of deformation, which shows a good tolerance of fabrication errors. 

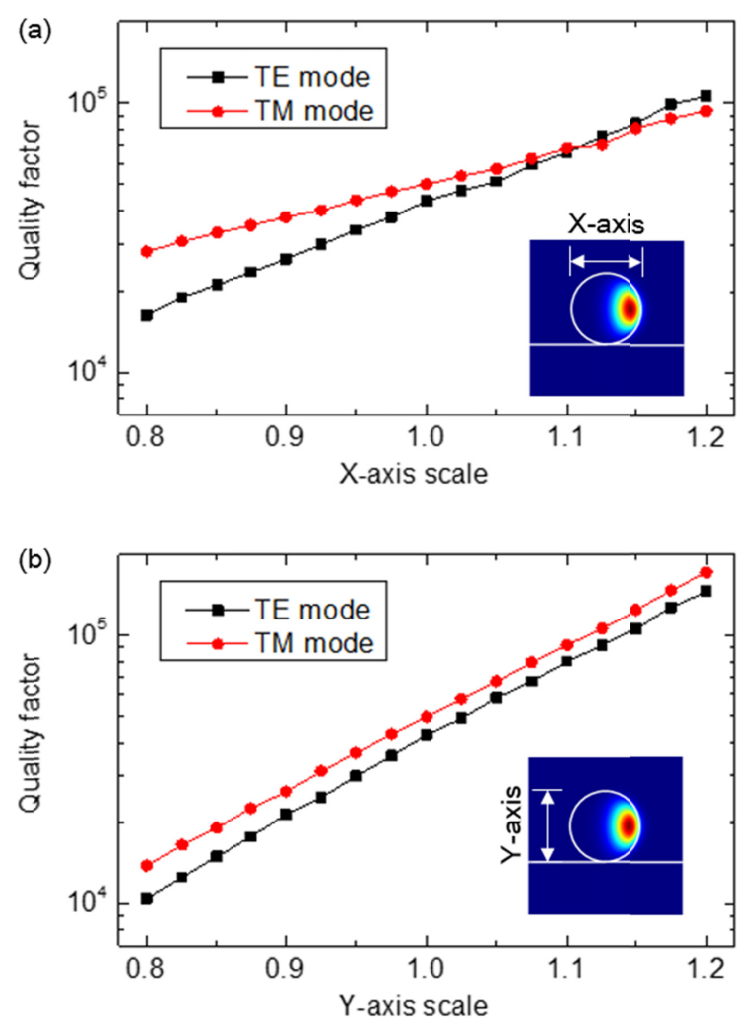

Fig. S7. Simulated $Q$ factors of fundamental TE (TM) mode versus cross-section geometry scale of X-axis (a) and Y-axis (b). The minor diameter of the original circular cross section is $6 \mu \mathrm{m}$. While one axis scale varies, the other one keeps unchanged. The principal diameter of the microcavity is $80 \mu \mathrm{m}$. 